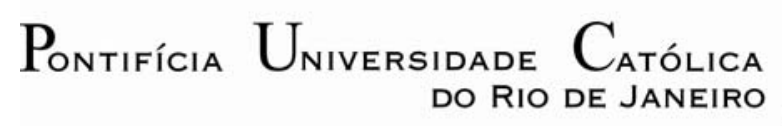

DO RIO DE JANEIRO

Jhoany Jhordann Barrera Escobedo

Modelagem Transiente da Transferência de Calor em
Dutos de Petróleo ou Gás, Termicamente Isolados

Dissertação de Mestrado

Dissertação apresentada como requisito parcial para obtenção do grau de Mestre pelo Programa de Pós-Graduação em Engenharia Mecânica da PUCRio.

Orientadora: Angela Ourivio Nieckele

Rio de Janeiro setembro de 2005 


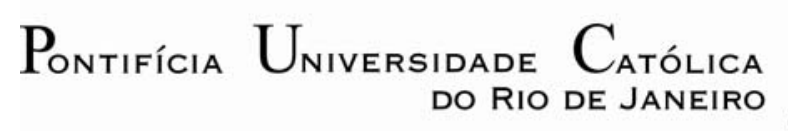

Jhoany Jhordann Barrera Escobedo

\section{Modelagem Transiente da Transferência de Calor em Dutos de Petróleo ou Gás, Termicamente Isolados}

Dissertação apresentada como requisito parcial para obtenção do grau de Mestre pelo Programa de PósGraduação em Engenharia Mecânica da PUC-Rio. Aprovada pela Comissão Examinadora abaixo assinada.

Prof ${ }^{\mathrm{a}}$. Angela Ourivio Nieckele Orientadora Departamento de Engenharia Mecânica - PUC-Rio

Prof. Luis Fernando Alzuguir Azevedo Co orientador Departamento de Engenharia Mecânica - PUC-Rio

Dr. Felipe Bastos de Freitas Rachid Universidade Federal Fluminense

Dr. Luis Fernando Gonçalves Pires Instituto de Pesquisa e Desenvolvimento - Ctex

Prof. José Eugenio Leal Coordenador Setorial do Centro Técnico Científico - PUC-Rio 
Todos os direitos reservados. É proibida a reprodução total ou parcial do trabalho sem autorização da universidade, do autor e do orientador.

Jhoany Jhordann Barrera Escobedo

Graduou-se em Engenharia Mecânica na Universidad Nacional de Ingeniería (Lima, Peru) em 2001.

Ficha Catalográfica

Barrera Escobedo, Jhoany Jhordann

Modelagem transiente da transferência de calor em dutos de petróleo ou gás, termicamente isolados / Jhoany Jhordann Barrera Escobedo; orientadora: Ângela Ourivio Nieckele; co-orientadora: Luis Fernando Alzuguir Azevedo. - Rio de Janeiro: PUC, Departamento de Engenharia Mecânica, 2005.

$125 \mathrm{f.} ; 30 \mathrm{~cm}$

Dissertação (mestrado) - Pontifícia Universidade Católica do Rio de Janeiro, Departamento de Engenharia Mecânica.

Inclui referências bibliográficas.

1. Engenharia mecânica - Teses. 2. Transiente térmico. 3. Resfriamento. 4. Linhas submarinas. 5. Capacidade térmica. I. Nieckele, Ângela Ourivio. II. Azevedo, Luis Fernando Alzuguir. III. Pontifícia Universidade Católica do Rio de Janeiro. Departamento de Engenharia Mecânica. IV. Título. 


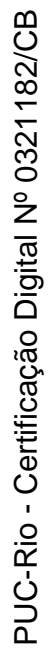

Para minha família 


\section{Agradecimentos}

À Professora Angela Ourivio Nieckele e ao Professor Luis Fernando Azevedo pela dedicada orientação no desenvolvimento desta dissertação e pelo apoio durante o curso de mestrado.

A minha família, em especial meus pais Orfelia e Hildomaro, e a minha irmã Magaly pelo apoio e estimulo durante este tempo longe de casa.

Aos amigos do SIMDUT, em especial ao Arturo Ortega e Cláudio Velosso pelas “dicas" e sugestões sobre os softwares comerciais de simulação de dutos.

Aos professores do Departamento de Engenharia Mecânica da PUC-Rio pelos conhecimentos fornecidos.

A todos os amigos do mestrado pelos bons momentos compartilhados.

A CAPES e à PUC-Rio pela ajuda financeira recebida durante o curso. 


\section{Resumo}

Barrera Escobedo, Jhoany Jhordann. Modelagem Transiente da Transferência de Calor em Dutos de Petróleo ou Gás, Termicamente Isolados. Rio de Janeiro, 2005, 125 p. Dissertação de Mestrado Departamento de Engenharia Mecânica, Pontifícia Universidade Católica do Rio de Janeiro.

Linhas submarinas são utilizadas na produção e transporte de petróleo e seus derivados. Em ambas as situações, o controle da transferência de calor do fluido para o ambiente externo pode ser um fator determinante para o escoamento. No caso de produção em águas profundas, o fluido aquecido perde calor para a água do mar gelada. A perda de calor é controlada através do isolamento térmico, o qual é projetado para operações de escoamento em regime permanente. Durante eventuais paradas de operação, o fluido estagnado no interior da tubulação ao perder calor para o ambiente frio, pode atingir níveis críticos de temperatura, acarretando graves problemas, tais como formação de hidratos ou deposição de parafina nas paredes da tubulação, o que pode levar ao bloqueio da linha e interrupção de produção. No transporte de produtos, o reinício de bombeio de fluidos muitos viscosos também é um problema crítico, devido ao aumento significativo da viscosidade com a redução da temperatura. O presente trabalho apresenta uma análise da influência da capacidade térmica da parede do tubo e das camadas de revestimento no transiente térmico de linhas com muito isolamento. A perda de calor da linha para o ambiente é determinada resolvendo-se a equação transiente de condução de calor para as camadas de revestimento da tubulação, utilizando um modelo uni-dimensional na direção radial. O método de volumes finitos é empregado para resolver o escoamento transiente no interior da tubulação acoplado com o transiente térmico na parede da tubulação, a partir do instante em que uma válvula é fechada na extremidade da tubulação interrompendo o fluxo. Comparações com as previsões de softwares comerciais foram realizadas e suas limitações são discutidas. Resultados obtidos das simulações para o escoamento tanto de líquidos quanto de gases, considerando e 
desprezando a capacidade térmica, mostram que o efeito da mesma é relevante na determinação do tempo de resfriamento da linha e do fluido em seu interior.

\section{Palavras-chave}

Transiente térmico, resfriamento, linhas submarinas, capacidade térmica. 


\section{Abstract}

Barrera Escobedo, Jhoany Jhordann. Transient Heat Transfer Modeling of Thermally Insulated Oil or Gas Pipelines. Rio de Janeiro, 2005, 125 p. MSc. Dissertation - Departamento de Engenharia Mecânica, Pontifícia Universidade Católica do Rio de Janeiro.

Subsea pipelines are employed not only for production but also for transportation. In both situations, warm oil loses heat to the cold sea water. The heat loss to the ambient is controlled by means of thermal insulation, which is designed for steady state operations. During shutdowns, the stagnant fluid in the pipeline loses heat to the cold surrounding, eventually reaching some critical temperature. As a result, several problems can occur, such as formation of hydrates or deposition of high molecular weight paraffins on the inner wall of the subsea line, which can lead to flow line blockage and production shutdown. Restart of very viscous fluid after shutdown is also critical, since viscosity increases significantly with the reduction of the temperature. This work presents an analysis of the influence of the pipe wall thermal capacitance on the transient behavior of heavily insulated lines. The heat loss from the pipeline is determined, by solving the transient heat conduction equation for the pipewall layers, utilizing a simple one-dimensional model in the radial direction. The finite volume method is employed to solve the transient flow inside the pipeline, from the time instant that a valve at the end point of the line is closed, coupled with the pipe wall thermal transient. Comparisons with the prediction of commercial softwares were performed and their limitations are addressed. Numerical results obtained for flows of both liquid and gases, considering and neglecting the thermal capacitance, revealed that accounting for the thermal capacity of the wall is relevant to the determination of cooldown times

\section{Keywords:}

Thermal model, transient, cooldown, sub-sea lines, heat capacity. 


\section{Sumário}

1. Introdução 19

1.1. Identificação do problema 19

1.2. Motivação 25

1.3. Revisão Bibliográfica 26

1.4. Objetivo 29

1.5. Organização do Trabalho 30

2. Modelamento Matemático 31

2.1. Conservação de Massa 32

2.1.1. Variações da Área da Tubulação 33

2.1.2. Compressibilidade do Fluido 34

2.1.3. Equação para a Pressão 34

2.2. Conservação da Quantidade de Movimento Linear 35

2.2.1. Fator de Atrito 36

2.3. Conservação da energia 37

2.3.1. Modelo Pigsim-W 40

2.3.2. Modelo Pigsim-U 42

2.3.3. Coeficientes convectivos de transferência de calor 43

2.4. Propriedades do Fluido 45

2.4.1. Massa Específica 46

2.4.2. Viscosidade Absoluta 47

2.5. Condições de Contorno 47

2.6. Condições Iniciais 48

2.6.1. Fluido em Repouso 48

2.6.2. Inicialização em Regime Permanente 49

2.7. Modelo Matemático utilizado pelo software Pipeline Studio 49

2.8. Modelo Matemático utilizado pelo software Stoner 54

2.9. Modelo Matemático utilizado pelo software OLGA 59 
3. Método Numérico 62

3.1. Discretização das Equações de conservação 62

3.1.1. Equação da Continuidade 64

3.1.2. Equação da Quantidade do Movimento Linear 65

3.1.3. Equação de conservação da energia 66

3.2. Discretização da equação de condução de Calor 67

3.3. Solução do Sistema Algébrico 69

3.4. Critério de Convergência 72

3.5. Diagrama de fluxo e procedimento geral 73

4. Análise de resultados 76

4.1. Escoamento em líquidos 78

4.1.1. Condições de contorno e inicial 81

4.1.2. Influência da capacidade térmica 82

4.1.3. Análise paramétrica 91

4.2. Escoamento de gás 98

4.2.1. Condições de contorno e inicial 99

4.2.2. Influência da capacidade térmica 101

4.2.3. Análise paramétrica 110

5. Comentários finais 113

$\begin{array}{ll}\text { Referências Bibliográficas } & 115\end{array}$

$\begin{array}{ll}\text { Apêndice A } & 118\end{array}$ 


\section{Lista de Figuras}

Figura 1.1 - Disposição da Máster-1 e da SDV em uma linha de produção.

Figura 1.2 - Bloqueio de linha por formação de hidratos

Figura 1.3 - Envelope de formação de hidratos

Figura 1.4 - Bloqueio de linha por formação de parafinas

22

Figura 1.5 - Arranjo Pipe-in-Pipe 23

Figura 1.6 - Arranjo Pipe-in-Pipe aquecido eletricamente.

Figura 2.1 - Volume de controle elementar

Figura 2.2 - Secção transversal utilizada pelo software OLGA 60

Figura 3.1 - Layout da tubulação 62

Figura 3.2 - Distribuição deslocada de malha

Figura 3.3 - Malha utilizada para a discretização da equação de condução de calor

Figura 3.4 - Esquemático do sistema algébrico heptadiagonal 69

Figura 3.5 - Fluxograma do modelo implementado

Figura 3.6 - Fluxograma da rotina MARCHA 75

Figura 4.1 - Configuração utilizada para o analise do transiente 76

Figura 4.2 - Regime permanente do teste do liquido

Figura 4.3 - Perfil de temperatura com o tempo em $x=500 \mathrm{~m}$.

Figura 4.4 - Perfil de temperatura após 4 horas do fechamento da válvula

Figura 4.5 - Perfil de temperatura após 8 horas do fechamento da válvula

Figura 4.6 - Perfis de temperatura ao longo do duto obtidos com o modelo Pigsim-W

Figura 4.7 - Temperatura na parede do duto em $x=500 \mathrm{~m}$.

Figura 4.8 - Perdas de calor em uma seção na metade do duto $(x=500 m)$

Figura 4.9 - Perfis de pressão e velocidade 
Figura 4.10 - Variação da pressão, velocidade e massa específica em $x=500 m$.

Figura 4.11 - Variação dos números de Reynolds e de Grashof com o tempo em $x=500 m$.

Figura 4.12 - Variação dos parâmetros $\operatorname{Re} \mathrm{Gr}$ e $\mathrm{Gr} / \mathrm{Re}^{2} \mathrm{com} 0$ tempo em $\mathrm{x}=500 \mathrm{~m}$.

Figura 4.13 - Variação do número de Nusselt com o tempo em x $=500 \mathrm{~m}$.

Figura 4.14 - Perfil de temperatura com o tempo em $x=500 m$. Avaliação da influência da condutividade térmica

Figura 4.15 - Perfil de temperatura com o tempo em $x=500 m$. Avaliação da influência da difusividade térmica

Figura 4.16 - Taxa de transferência de calor com o tempo em uma seção na metade do duto $(\mathrm{x}=500 \mathrm{~m}) \cdot \alpha_{i s o} / \alpha=\infty$

Figura 4.17 - Taxa de transferência de calor com o tempo em uma seção na metade do duto $(\mathrm{x}=500 \mathrm{~m}) \cdot \alpha_{i s o} / \alpha$ $=\infty$ e $\alpha_{a c o} / \alpha=\infty$

Figura 4.18 - Variação da temperatura com o tempo em $\mathrm{x}=$ 500m. Avaliação da influência da espessura do isolamento

Figura 4.19 - Variação da temperatura com o tempo em $\mathrm{x}=$ 500m. Avaliação da influência do diâmetro do duto.

Figura 4.20 - Regime permanente para o escoamento do gás 100

Figura 4.21- Variação da temperatura com o tempo em $x=10$ $\mathrm{km}$

Figura 4.22- Perfil de temperatura após 2 horas do fechamento da válvula

Figura 4.23- Perfil de temperatura após 4 horas do fechamento da válvula

Figura 4.24- Perfis de temperatura obtidos com o modelo Pigsim-W

Figura 4.25- Variação da temperatura da parede do duto com o tempo em $\mathrm{x}=10 \mathrm{~km}$ 
Figura 4.26- Perdas de calor em uma seção na metade do duto $(\mathrm{x}=10 \mathrm{~km})$

Figura 4.27- Perfis de pressão e velocidade

Figura 4.28- Variação da velocidade, massa específica e pressão com o tempo em $\mathrm{x}=10 \mathrm{~km}$.

Figura 4.29- Variação do número de Reynolds com o tempo em $x=10 \mathrm{~km}$.

Figura 4.30- Variação temporal do número de Grashof e da diferença de temperaturas entre o fluido e a parede interna do duto $\left(T-T_{\text {sol }}\right)$ em $x=10 \mathrm{~km}$.

Figura 4.31- Variação temporal dos parâmetros $\operatorname{ReGr}$ e $\mathrm{Gr} / \mathrm{Re}^{2}$ em $x=10 \mathrm{~km}$.

Figura 4.32- Variação temporal dos números de Reynolds e Nusselt em $x=10 \mathrm{~km}$.

Figura 4.33- Perfis de temperatura com o tempo em $x=10 \mathrm{~km}$. Avaliação da difusividade térmica.

Figura 4.34 - Comparação das correlações utilizadas pelos diferentes softwares

Figura A.1- Comparação da solução numérica com a solução exata 120

Figura A.2- Erros normalizados utilizando esquemas de discretização linear (LIN) e logarítmico (LOG) para diferentes passos de tempo, NC $=5$

Figura A.3- Erros normalizados utilizando esquemas de discretização linear (LIN) e logarítmico (LOG) para diferentes passos de tempo, $\mathrm{NC}=50$

Figura A.4- Solução do regime transiente para diferentes passos de tempo, $\mathrm{NC}=5$

Figura A.5- Temperaturas em regime permanente: $\mathrm{NC}=5, \mathrm{Dt}=10 \mathrm{~s}$

Figura A.6- Solução em regime transiente para diferentes passos de tempo, $\mathrm{Nc}=5$

Figura A.7- Influência do número de volumes de controle (V.C.) por camada 


\section{Lista de tabelas}

Tabela 4.1 - Propriedades da parede do duto

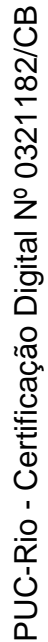




\section{Nomenclatura}

$a \quad$ Velocidade do som $(\mathrm{m} / \mathrm{s})$

$a_{0}, a_{1}, a_{2}$, Coeficientes de ajuste da equação do calor específico

$a_{3}, a_{4}, a_{5}$ Coeficientes de ajuste da equação do calor específico

$A \quad$ Área da seção transversal

$A, B, C, D$ Constantes empíricas da correlação de Standing \& Katz

$A_{g} \quad$ Área de passagem da válvula

$c_{p} \quad$ Calor específico a pressão constante.

$c_{p, s} \quad$ Calor específico do material da camada

$c_{\mu, p} \quad$ Coeficiente de pressão da viscosidade

$c_{\mu, T} \quad$ Coeficiente de temperatura da viscosidade

$c_{v, \text { ref }} \quad$ Calor específico a volume constante do software Stoner

$C_{c v, T} \quad$ Dependência do calor específico com a temperatura.

$C_{k, T} \quad$ Dependência da condutividade térmica com a temperatura.

$C_{D} \quad$ Coeficiente de descarga

D Diâmetro do tubo (m)

$D_{e} \quad$ Diâmetro externo do tubo (m)

$D_{\text {ref }} \quad$ Diâmetro do tubo à pressão atmosférica (m)

$D_{r e v, j} \quad$ Diâmetro exterior da camada $j$

$D_{o} \quad$ Diâmetro externo da tubulação (m)

$D_{1}, D_{2}, D_{3}$ Coeficientes do calculo da viscosidade da equação de Lee- e GonzalesEakin

$e \quad$ Espessura da parede do tudo (m)

$e \quad$ Energia total específica

$e_{j} \quad$ Espessura da camada $j$

E $\quad$ Modulo de Young

$f_{a t} \quad$ Fator de atrito

F $\quad$ Força

$g \quad$ Aceleração da gravidade $\left(\mathrm{m} / \mathrm{s}^{2}\right)$ 





$\mathrm{Re}_{1}, \mathrm{Re}_{2}$ Parâmetros para caracterizar o escoamento laminar o turbulento no software Stoner

$\operatorname{Res}_{j} \quad$ Resistência térmica equivalente da parede do tubo

$\Re \quad$ Constante universal dos gases $(\mathrm{J} / \mathrm{kgmol} \cdot \mathrm{K})$

$S G \quad$ Gravidade especifica

$t \quad$ Tempo (s)

tol $_{1} \quad$ Tolerância no cálculo do erro absoluto

$t_{2} \quad$ Tolerância no cálculo do erro normalizado

$T \quad$ Temperatura do fluido

$T_{c r} \quad$ Temperatura crítica

$T_{\text {crm }} \quad$ Temperatura pseudo-crítica (K)

$T_{\text {ref }} \quad$ Temperatura de referencia $(\mathrm{K})$

$\operatorname{Tr} \quad$ Temperatura reduzida,

$T_{s, j} \quad$ Temperatura da camada $(\mathrm{K})$

$T_{s, 1} \quad$ Temperatura da primeira camada (parede do duto) (K)

$T_{S,} \quad$ Temperatura da última camada de revestimento $(\mathrm{K})$

$T_{w} \quad$ Temperatura da parede interna da tubulação (K)

$T_{\infty} \quad$ Temperatura do ambiente externo (K)

$u \quad$ Energia específica interna

$U_{e} \quad$ Coeficiente global de troca térmica $\left(\mathrm{W} /\left(\mathrm{m}^{2} \mathrm{~K}\right)\right)$

$V \quad$ Velocidade $(\mathrm{m} / \mathrm{s})$

$V_{\infty} \quad$ Velocidade da corrente externa $(\mathrm{m} / \mathrm{s})$

$V_{t} \quad$ Velocidade de transferência de massa

$\dot{W} \quad$ Trabalho realizado no volume de controle.

$\dot{W}_{p} \quad$ Taxa de trabalho devido às forças de pressão

$\dot{W}_{g} \quad$ Taxa de trabalho devido às forças gravitacionais

$x \quad$ Coordenada axial

$z \quad$ Elevação $(\mathrm{m})$

$z \quad$ Fator de compressibilidade

\section{Letras Gregas:}
$\alpha \quad$ Fração volumétrica de gás
$\alpha_{s, j} \quad$ Difusividade térmica do material da camada 
$\beta \quad$ Coeficiente de expansão térmica

$\beta_{\text {ref }} \quad$ Coeficiente de expansão térmica de referencia

$\Delta T \quad$ Diferença de temperaturas entre o fluido e a parede interna.

$\chi \quad$ Percentagem de abertura da válvula

$\varphi \quad$ Parâmetro que controla o uso da correlação do Nusselt do software Stoner

$\varepsilon \quad$ Rugosidade do tubo (m)

$\mu \quad$ Coeficiente de Poisson

$\mu_{f} \quad$ Viscosidade absoluta do fluido $(\mathrm{kg} / \mathrm{m} \cdot \mathrm{s})$

$\mu_{\infty} \quad$ Viscosidade absoluta do ambiente marinho $(\mathrm{kg} / \mathrm{m} \cdot \mathrm{s})$

$\mu_{m} \quad$ Viscosidade da película do fluido junto à parede

$\mu_{\text {ref }} \quad$ Viscosidade absoluta de referência

$\theta \quad$ Ângulo do eixo do tubo com a horizontal

$\rho \quad$ Massa específica $\left(\mathrm{kg} / \mathrm{m}^{3}\right)$

$\rho_{\text {ref }} \quad$ Massa específica $\left(\mathrm{kg} / \mathrm{m}^{3}\right)$

$\rho_{s, j} \quad$ Massa especifica da camada $\left(\mathrm{kg} / \mathrm{m}^{3}\right)$

$\rho_{\infty} \quad$ Densidade do ambiente marinho $\left(\mathrm{kg} / \mathrm{m}^{3}\right)$

$\tau_{s} \quad$ Tensão cisalhante

$\tau_{i} \quad$ Tensão cisalhante na interface líquido-gás

$\tau_{w g} \quad$ Tensão cisalhante da fase gasosa com a parede da tubulação

$\tau_{w l} \quad$ Tensão cisalhante da fase liquida com a parede da tubulação

$\Gamma_{g} \quad$ Troca de massa entre as fases gás e liquido

\section{Subscritos:}

$\begin{array}{ll}i & \text { Ponto nodal } \\ \text { in } & \text { Entrada do duto } \\ m & \text { Média } \\ \text { out } & \text { Saída do duto } \\ \text { ref } & \text { Variáveis de referência }\end{array}$

\section{Sobrescritos:}

o Instante do tempo anterior 